\title{
Evaluation of Phase Shift in Electric Drive by Kalman Filter for Wheel Slip Control
}

\author{
Petr Pichlík \\ Dept. of Electric Drives and Traction \\ Czech Technical University in Prague, Faculty of Electrical Engineering \\ Technická 2, 16627 Prague, Czech Republic \\ pichlpet@fel.cvut.cz
}

\begin{abstract}
Slip controller is an essential part of the locomotive controller because it enables maximum force transfer and increases the service life of the locomotive parts. The modern slip controllers try to determine actual adhesion parameters and set the required tractive force to avoid slippage occurrence. Many types of the slip controller methods were developed, and one of developed principle try to determine adhesion parameters from phase shift between motor torque and corresponding speed measured on the motor or wheelset. The paper presents a novel evaluation procedure for the phase shift calculation that is based on the Kalman filter that enables directly compare force corresponding with the motor torque and the adhesion force. The method principle is described in the paper, and the method performance is evaluated on the mathematical model.
\end{abstract}

Keywords-adhesion; slip control; railway transport; Kalman filter.

\section{INTRODUCTION}

Locomotives are equipped by slip controllers to be ensured high tractive force transmission from wheels to rails, and simultaneously the wheel slip velocity is kept at the acceptable value. The problem with the force transfer is most significant in trains that are hauled by locomotive especially in freight trains. If the slip controller does not work correctly, high wear of wheels and rails occur [1], some mechanical part can be damaged, the train can have delay [2], or the train can be jamming on the track in the worst case. The problem with the adhesion is caused by an adhesion coefficient permanent change [3]. The maximum transferable force depends on the actual adhesion coefficient value. When the applied force to the wheelset is higher than the maximum transferable force, the wheels start accelerates. The properly worked slip controller should avoid the slippage creation by a preventive decrease of the applied force according to the actual adhesion conditions.

The developed slip control methods can be divided into re-adhesion controllers and slip controllers [4]. The re-adhesion controllers are outdated because they cannot prevent the slippage occurrence, but they limit the created slippage only. The effective slip controllers can be divided into methods that try to determine the maximum transferable force value and methods that try to determine a slope of actual adhesion characteristic in the operating point. The methods that try to determine the maximum transferable force increases applied force until the maximum force is not exceeded and then decreases the applied force to avoid slippage and keep the applied force near the maximum transferable force [5]. The method advantage is that it can fully utilise the available adhesion and the method does not need the actual value of the slip velocity for its proper work. That is a high benefit of the method because the slip velocity determination is not easy when all wheelsets are driven [6]. On the other hand, the method is not appropriate because it produces undesirable effects like slick-clip oscillations that occur during the maximum force searching [7]. The slip control methods that are based on an adhesion characteristic slope determination seems like the most effective from the developed method during the last decades. Because they can reach almost the maximum point on the characteristic without the maximum point overstep. The adhesion characteristic slope can be determined by using some estimators to determine adhesion coefficient [8] or by determination of a phase shift of injected excitation signal between motor and wheels [9]. The determination of the phase shift between motor and wheels requires a dynamic electric drive with high-quality torque control to be possible injects the signal [10]. The evaluation of the phase shift from measured wheelset velocity is also a difficult task because the measurement is noisy, resolution of measurement depends on the actual velocity and the signal can be highly damped and its loss can be a result. Therefore, the signal has to be filtered to remove the noise and DC offset. The DC offset has to be removed to be possible to calculate the phase shift.

The paper presents an improvement of the phase shift evaluation by using a Kalman filter (KF). The KF is used for estimation of adhesion force that is used for comparison with motor torque. The KF improves filtration, solve the problem with DC offset and can improve method performance when high damping occurs in the axle mechanical part that can cause the signal loss. The KF improved this state by estimation of the damped signal. The rest of the paper is organised as follows. In part II the method fundamental principles, phase calculation and the KF algorithm and its properties are described. In part III are presented simulation results. 


\section{METHOD PRINCIPLE}

The force between wheels and rails is transferred through adhesion. The adhesion is typically depicted as a dependence of a slip velocity. Therefore, the principle of all modern slip controllers has to be based on the adhesion characteristic shape. The example of the characteristic is depicted in Fig. 1. The characteristic can be divided into a stable and unstable part from the electric point of view. If the operating point is located in the stable area, the electric drive can stably work in the area without any slip controller reaction. When the operating point gets to the unstable area, the electric drive speed starts to increase. This situation has to be limited by the slip controller reaction or in better case the situation should be avoided by the slip controller preventively action. The slip controller can limit or avoid the situation by decreasing the motor torque. The motor torque has to be decreased in the right moment. Otherwise, the slippage can occur if the torque is decreased lately or the adhesion utilisation is bad if the torque is decreased before the appointed time. So, the torque should be decreased when the operation point is close to the maximum point in a stable area. To ensure the requirement for the slip controller correct operation is complicated because the characteristic shape continually changes during the train run and locate the operating point near the maximum point requires accurate slip controller and an electric drive with a high dynamic. If the requirements to the slip controller and an electric drive are not met, the operating point has to be located further of the maximum point. The adhesion utilisation is worse in the time.

The proposed method is based on the estimation of the phase shift between the required torque of the electric drive and measured revolutions of the motor. The method adds a low-frequency excitation signal to the required motor torque. The excitation signal amplitude can be up to $10 \%$, and the frequency has to be lower than the lowest eigenfrequencies of the mechanical part of axle [10]. Therefore, the frequency should be around $10 \mathrm{~Hz}$. The frequency should be as high as possible because the frequency depends on the detection delay.

The phase shift in the electric drive and connected mechanical parts can be determined because when the operating point is located in the stable area of the adhesion characteristic, the dynamic effects are damped and when the operating point is located in the unstable area the dynamic motions are damped negatively. The required motor torque has to contain an excitation signal that enables to determine the phase shift. The signal from the required motor torque is simple to determine because it is calculated by the axle computer. However, the signal from the motor revolution is difficult to obtain in a necessary quality. There are several reasons for the signal disturbance. The main problem is the excitation signal disappearance due to the wheelset mechanical damping. The method principle with the signals is schematically depicted in Fig. 2. The schema consists of the electric drive computer, electric drive and axle mechanical parts. There are two points in which are get two signals that are compared between them in Fig. 2.

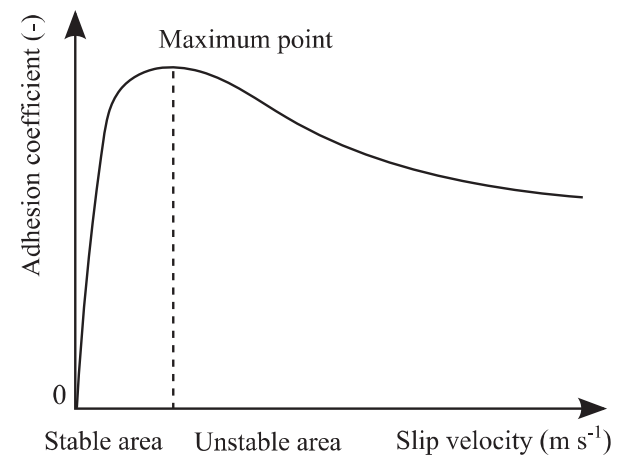

Figure 1. Example of the adhesion characteristic for good adhesion conditions

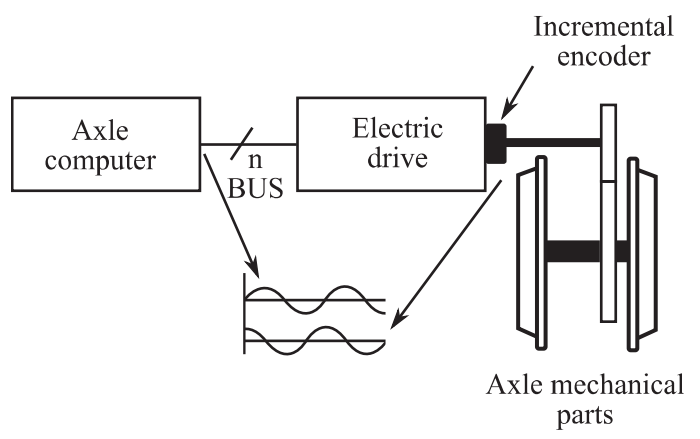

Figure 2. Method general principle

\section{A. Phase Shift Calculation}

The phase shift calculation is based on a simple principle that uses multiplication of two general vectors with a phase shift:

$$
\begin{gathered}
a_{n}=\sin (n \omega) \\
b_{n}=\cos (n \omega+\varphi)
\end{gathered}
$$

Where $a$ and $b$ are vectors of length $N, \omega$ is a signal frequency.

Vectors $a$ and $b$ have to capture the whole period of the excitation signal, and the length of the vectors is given by the excitation signal frequency and sampling period that should correspond with the control loop period. The main task of the method is to get data from vectors $a$ and $b$ fill. To get a clear signal from the required torque is simple because the method requires information about the period beginning only. Therefore, this part can be replaced by a sinus signal. To get signal from the speed sensor is more difficult and the original method requires band-pass and lowpass filters to remove noise. The filters have to be higher order that increases the required calculation time. The final phase shift is calculated as follows:

$$
\cos \varphi=\frac{\sum_{n=0}^{N} a_{n} b_{n}}{\sqrt{\sum_{n=0}^{N} a_{n}^{2}} \sqrt{\sum_{n=0}^{N} a_{n}^{2}}}
$$


The calculated phase shift expresses the phase shift that is caused by the adhesion change that influences the mechanical part of the axle but the phase shift as it is depicted in Fig. 2 is also influenced by the electric drive control. Moreover, the delay cussed by the filtration of the velocity signal has to be also considered. Therefore, the signal with the excitation signal has to be also delayed, or the phase shift caused by the different timing of the individual signals has to be taken into account.

The speed signal is filtered by a KF to get the correct representation of the excitation signal. The KF is used because it can filter the proper value more precise than the conventional filters and can the signal estimate. Moreover, KF implementation requires less computational time than conventional filters. The well-known KF algorithm is used [11]:

$$
\begin{gathered}
K=\frac{P_{k} H_{k}^{T}}{H_{k} P_{k} H_{k}^{T}+R} \\
\hat{x}_{k}=\hat{x}_{k}+K\left(z_{k}-H_{k} \hat{x}_{k}\right) \\
P_{k}=\left(I-K_{k} H_{k}\right) P_{k} \\
\hat{x}_{k}=\phi_{k-1} \hat{x}_{k-1} \\
P_{k}=\phi_{k-1} P_{k-1} \phi_{k-1}^{T}+Q_{k-1}
\end{gathered}
$$

where $\boldsymbol{K}$ is the Kalman gain matrix, $\boldsymbol{P}$ is state estimation uncertainty covariance matrix, $\boldsymbol{H}$ is measurement sensitivity matrix, $\boldsymbol{R}$ is measurement uncertainty covariance matrix, $\boldsymbol{x}_{\mathrm{h}}$ is system state vector, $z_{\mathrm{n}}$ are measured values, $\phi$ is system matrix in discrete time, and $\boldsymbol{Q}$ is process noise covariance matrix.

The mathematical model that is used by the KF is a two-mass model. The mode is created by motor mas and the wheel mass that are connected by an elastic element. The model features are described in [12] in detail. The model state matrix in continues time and system state vector are defined as follows:

$$
A=\left[\begin{array}{cccc}
0 & \frac{1}{J_{1}} & \frac{1}{J_{2}} & 0 \\
c_{12} & -d_{12} & d_{12} & 0 \\
c_{12} & d_{12} & -d_{12} & -\frac{1}{J_{2}} \\
0 & 0 & 0 & -\frac{1}{T}
\end{array}\right]
$$

$$
x=\left[\begin{array}{llll}
\Delta \gamma & v_{M} & v_{W} & F_{A}
\end{array}\right]^{T}
$$

Where $J_{1}$ and $J_{2}$ are moments of inertia of motor and wheel respectively, $c_{12}$ is stiffness between masses, $d_{12}$ is damping between masses, $T$ is an electric drive time constant, $\Delta \gamma$ is swivelling angle between motor and wheelset, $v_{\mathrm{M}}$ and $v_{\mathrm{W}}$ are velocities of motor and wheelset respectively, and $F_{\mathrm{A}}$ is estimated adhesion force.

The original method compares the torque signal with the velocity signal for the phase shift calculation. From the state vector, it is clear that the KF can estimate an adhesion force. Therefore, a direct comparison of the force signal calculated from the required torque with the adhesion force is possible. This approach advantage is that the additional filters are not needed for a DC offset removing because the estimated adhesion force can be calculated without DC offset as a relative force by the KF. The block diagram of the proposed slip control method is depicted in Fig. 3. The method uses a different signal than the original method depicted in Fig. 2. The process of the excitation signal requires a time delay that corresponds to the KF time delay only. The speed signal is processed by the KF, and the estimated adhesion force is used instead of the speed. The dotted lines that connect the estimated adhesion force with wheel-rail contact illustrate the meaning of the adhesion force. The excitation signal is used by the KF for the excitation signal improvement only.

\section{Simulation ReSUlts}

The proposed phase calculation method was simulated on a locomotive mathematical model that represents one axle. The one axle model is used because every axle has independent slip controller. This approach was chosen because locomotive computers are designed as distributed computers [13] that cannot provide all information in real time between the local computers. The simulation results are depicted in Fig. 4 and Fig. 5.

The simulated wheelset velocity, train velocity, tractive force and adhesion force is depicted in Fig. 4. The adhesion force starts to decrease a time 1.4 second. The decrease is caused by, e.g. some contaminations on the surface of the rails. The tractive force is the same for the whole simulation. Therefore, a slippage occurs as it is shown in the wheelset velocity time course. The adhesion starts to decrease at time 1.4, but the maximum point is overstepped at time 1.7. Therefore, till the time there is no slippage but the slip velocity increase only. The excitation signal is clearly visible in the tractive force, but the excitation signal influence to the wheelset velocity is less significant. The amplitude of the excitation signal added to the force is $2.5 \%$ of the actual force value. The amplitude of the excitation signal in the wheelset velocity is $0.1 \%$ of the actual velocity value only. 


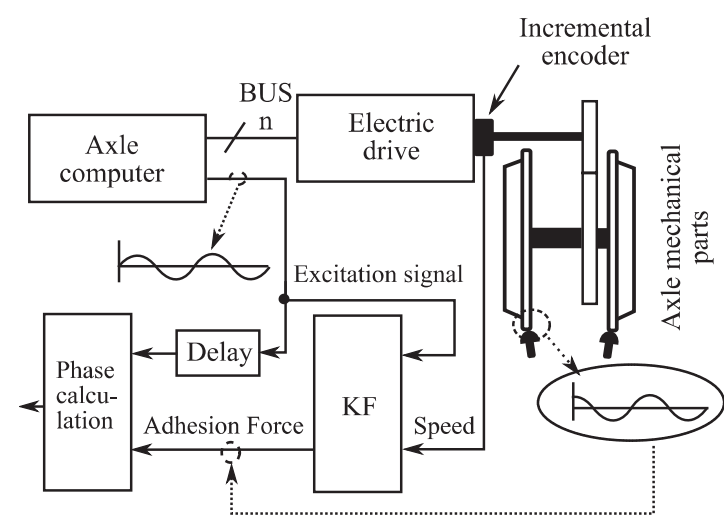

Figure 3. Block diagram of the proposed slip control method
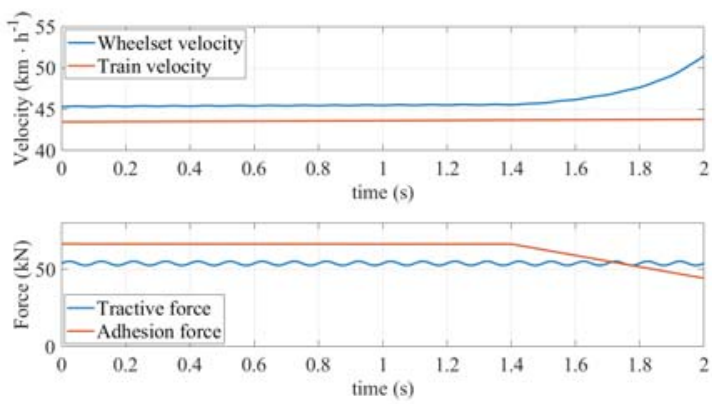

Figure 4. Velocities and forces
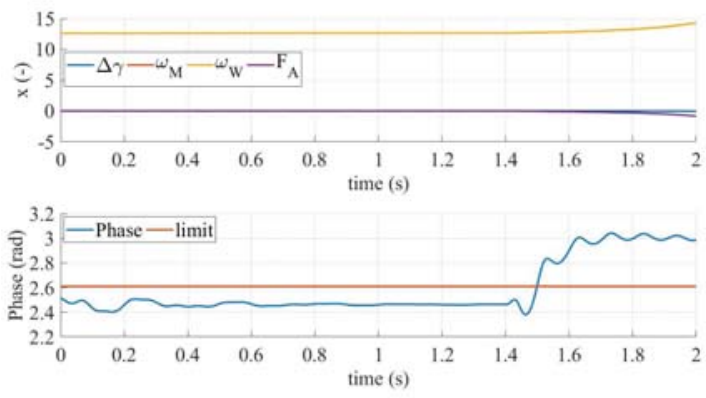

Figure 5. State vector and calculated phase

The state vector of the KF and calculated phase is depicted in Fig. 5. The phase is calculated from the state variable $F_{\mathrm{A}}$ that is the adhesion force. The amplitude of the excitation signal that appears at the state variable is $50 \%$. The signal with this amplitude is better for comparison than the velocity signal. The calculated phase is almost constant when there is no slippage, and its value is below $2.6 \mathrm{rad}$. When the slip velocity starts to increase, the phase starts to rise. When the slippage occurs, the value is around $3.1 \mathrm{rad}$.

\section{CONCLUSIONS}

The paper describes a novel method for the phase shift calculation between excitation signal injected to the required motor torque and signal that can be measured on the wheelset. The calculated phase shift can be used for wheel slip control purposes. The improvement of the method leads in the usage of the
$\mathrm{KF}$ that estimates the adhesion force that can be directly used for the phase shift without any additional filtration, especially removing of a DC offset from the measured velocity. The estimated adhesion force can be directly used because the DC offset is removed by considering of the tractive force as a noise. The method was evaluated on a simplified locomotive model that is created by one axle model in open-loop. The phase change is significant when the slippage occurs as it is shown on simulation results.

\section{ACKNOWLEDGMENT}

This work was supported by the Technology Agency of the Czech Republic under the grant No. TE02000103, Center for Intelligent Drives and Advanced Machine Control.

\section{REFERENCES}

[1] I. Hussain, T. X. Mei, and R. T. Ritchings, "Estimation of wheel-rail contact conditions and adhesion using the multiple model approach," Vehicle System Dynamics, vol. 51, pp. 32 53, Jan. 2013

[2] X. Cao et al., "The effect of alumina particle on improving adhesion and wear damage of wheel/rail under wet conditions", Wear, vol. 348-349, pp. 98-115, Feb. 2016.

[3] H. Chen, T. Ban, M. Ishida, T. Nakahara, "Experimental instigation of influential factors on adhesion between wheel and rail under wet conditions", Wear, vol. 265, no 9-10, 2008, pp. 1504-1511.

[4] K. Xu, G. Xu, and C. Zheng, 'Novel determination of WheelRail adhesion stability for electric locomotives', Int. J. Precis. Eng. Manuf., vol. 16, no. 4, pp. 653-660, Apr. 2015.

[5] S. Sadr, D. A. Khaburi and J. Rodríguez, "Predictive Slip Control for Electrical Trains," in IEEE Transactions on Industrial Electronics, vol. 63, no. 6, pp. 3446-3457, June 2016.

[6] P. Pichlik and J. Zdenek, "Locomotive velocity estimation for a slip control purpose by an unscented Kalman filter," 2017 18th International Scientific Conference on Electric Power Engineering (EPE), Kouty nad Desnou, 2017, pp. 1-5.

[7] R. Stock, L. Stanlake, C. Hardwick, M. Yu, D. Eadie, R. Lewis, "Material concepts for top of rail friction management - Classification, characterisation and application" Wear, vol. 366-367, 2016, pp. 225-232.

[8] Y. Shimizu, K. Ohishi, T. Sano, S. Yasukawa and T. Koseki, "Anti-slip re-adhesion control based on disturbance observer considering bogie vibration," 2007 European Conference on Power Electronics and Applications, Aalborg, 2007, pp. 1-10.

[9] X. Hong, R. Zhang, L. Wu, Y. Li and K. Wang, "Simulation of adhesion control method based on phase-shift," 2013 International Conference on Electrical Machines and Systems (ICEMS), Busan, 2013, pp. 2077-2080.

[10] P. Karlovsky and J. Bauer, "Wheel Slip Determination Capability of Locomotive Driven by Model Predictive Control," 2018 IEEE 18th International Power Electronics and Motion Control Conference (PEMC), Budapest, 2018.

[11] M. S. Grewal, A. P. Andrews, Kalman Filtering Theory and Practice Using MATLAB. 3rd edition, Wiley, New York 2008.

[12] P. Pichlík and J. Zděnek, "Locomotive Wheel Slip Control Method Based on an Unscented Kalman Filter", IEEE Transactions on Vehicular Technology, 2018

[13] J. Zdenek, "Distributed control computer backbone communication channel of electric locomotive with effective DMA support," Proceedings of 14th International Power Electronics and Motion Control Conference EPE-PEMC 2010, Ohrid, 2010, pp. T6-27-T6-34. 